\title{
A Novel PCA-Based Bayes Classifier and Face Analysis
}

\author{
Zhong Jin ${ }^{1,2}$, Franck Davoine ${ }^{3}$, Zhen Lou ${ }^{2}$, and Jingyu Yang ${ }^{2}$ \\ 1 Centre de Visió per Computador, \\ Universitat Autònoma de Barcelona, Barcelona, Spain \\ zhong.jin@cvc.uab.es \\ 2 Department of Computer Science, \\ Nanjing University of Science and Technology, \\ Nanjing, People's Republic of China \\ jyyang@mail.njust .edu.cn \\ 3 HEUDIASYC - CNRS Mixed Research Unit, \\ Compiègne University of Technology, \\ 60205 Compiègne cedex, France \\ franck.davoine@hds.utc.fr
}

\begin{abstract}
The classical Bayes classifier plays an important role in the field of pattern recognition. Usually, it is not easy to use a Bayes classifier for pattern recognition problems in high dimensional spaces. This paper proposes a novel PCA-based Bayes classifier for pattern recognition problems in high dimensional spaces. Experiments for face analysis have been performed on CMU facial expression image database. It is shown that the PCA-based Bayes classifier can perform much better than the minimum distance classifier. And, with the PCA-based Bayes classifier, we can obtain a better understanding of data.
\end{abstract}

\section{Introduction}

In recent years, many approaches have been brought to bear on pattern recognition problems in high dimensional space. Such high-dimensional problems occur frequently in many applications, including face recognition, facial expression analysis, handwritten numeral recognition, information retrieval, and contentbased image retrieval. The main approach applies an intermediate dimension reduction method, such as principal component analysis (PCA), to extract important components for linear discriminant analysis (LDA) [1,2].

PCA is a classical, effective and efficient data representation technique. It involves a mathematical procedure that transforms a number of (possibly) correlated variables into a (smaller) number of uncorrelated variables called principal components.

The classical Bayes classifier plays an important role in statistical pattern recognition. Usually, it is not easy to use a Bayes classifier for pattern recognition problems in high dimensional space. The difficulty is in solving the singularity of covariance matrices since pattern recognition problems in high dimensional spaces are usually so-called undersampled problems. 
In this paper, we seek a PCA-based Bayes classifier by combining PCA technique and Bayesian decision theory. It is organized as follows. Section 2 gives an introduction to Bayesian decision theory. A PCA-based Bayes classifier is proposed in Section 3. Experiments for face analysis are performed in Section 4. Finally, conclusions are given in Section 5 .

\section{Bayesian Decision Theory}

Bayesian decision theory is fundamental in statistical pattern recognition.

\subsection{Minimum-Error-Rate Rule}

Let $\left\{\omega_{1}, \cdots, \omega_{c}\right\}$ be the finite set of $c$ states of nature ("categories"). Let the feature vector $x$ be a $d$-dimensional vector-values random variable and let $p\left(x \mid \omega_{j}\right)$ be the state-conditional probability density function for $x$, with the probability density function for $x$ conditioned on $\omega_{j}$ being the true state of nature. Let $P\left(\omega_{j}\right)$ describe the prior probability that nature is in state $\omega_{j}$. The target is to make a decision for the true state of nature.

It is natural to seek a decision rule that minimizes the probability of error, that is, the error rate. The Bayes decision rule to minimize the average probability error calls for making a decision that maximizes the posterior probability $P\left(\omega_{i} \mid x\right)$. It can formally be written as the argument $i$ that maximizes the posterior probability $P\left(\omega_{i} \mid x\right)$, that is,

$$
x \rightarrow \omega_{i} \text { with } i=\arg \max _{j} P\left(\omega_{j} \mid x\right) .
$$

The structure of a Bayes classifier is determined by the conditional densities $p\left(x \mid \omega_{j}\right)$ as well as by the prior probabilities $P\left(\omega_{j}\right)$. Under the assumption of the same prior probabilities $P\left(\omega_{j}\right)(j=1, \cdots, c)$ for all the $c$ classes, the minimumerror-rate rule of Eq. (11) can be achieved by use of the state-conditional probability density function $p\left(x \mid \omega_{j}\right)$ as follows

$$
x \rightarrow \omega_{i} \text { with } i=\arg \max _{j} p\left(x \mid \omega_{j}\right) .
$$

Of the various density functions that have been investigated, none has received more attention than the multivariate normal or Gaussian density. In this paper, it is assumed that $p\left(x \mid \omega_{j}\right)$ is a multivariate normal density in $d$ dimensions as follows

$$
p\left(x \mid \omega_{j}\right)=\frac{1}{(2 \pi)^{d / 2}\left|\Sigma_{j}\right|^{1 / 2}} \exp \left[-\frac{1}{2}\left(x-\mu_{j}\right)^{t} \Sigma_{j}^{-1}\left(x-\mu_{j}\right)\right],
$$

where $\mu_{j}$ is the $d$-component mean vector and $\Sigma_{j}$ is the $d \times d$ covariance matrix.

\subsection{Minimum Distance Classifier}

The simplest case occurs when the features are statistically independent and when each feature has the same variance, $\sigma^{2}$. In this case, the covariance matrix 
is diagonal, being merely $\sigma^{2}$ times the identity matrix $I$, that is

$$
\Sigma_{j}=\sigma^{2} \mathrm{I} \quad(j=1, \cdots, c) .
$$

Thus, the minimum-error-rate rule of Eqs. (2), (3) and (4) can be expressed as follows

$$
x \rightarrow \omega_{i} \text { with } i=\arg \min _{j}\left\|x-\mu_{j}\right\|^{2},
$$

where $\|\cdot\|$ denotes the Euclidean norm. This is the commonly used minimum distance classifier.

\subsection{Limitation of Bayes Classifier}

In a high dimensional space, some classes may lie on or near a low dimensional manifold. In other words, for some classes, the covariance matrices $\Sigma_{j}$ may be singular in high dimensional space. Such a limitation exists even in 2-dimensional spaces. A two-class problem is shown in Fig. 1]. In the example, one class de-

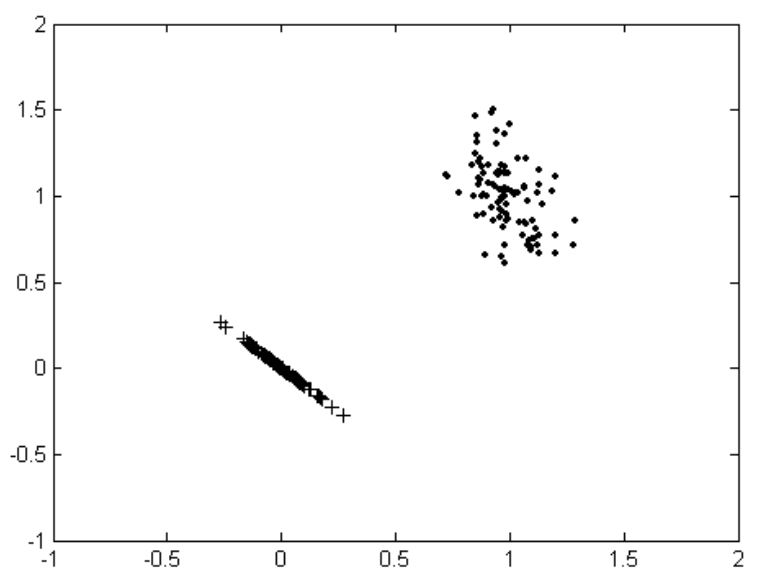

Fig. 1. A two-class problem

generates in a 1-dimensional line so that the Bayes classifier can not directly be used to perform classification. Anyway, the minimum distance classifier can be used to perform classifications. However, we fail to have a correct understanding of data since the constraint of Eq. (4) is not satisfied in the two-class problem.

\section{PCA-Based Bayes Classifier}

One solution to the above limitation of Bayes classifier is to describe Gaussian density of Eq. (3) by using principal component analysis (PCA). We are going to propose a novel PCA-based Bayes classifier in this section. 


\subsection{PCA Model}

Let $\Psi_{j}=\left(\psi_{j 1}, \cdots, \psi_{j d}\right)$ be the matrix whose columns are the unit-norm eigenvectors of the covariance matrix $\Sigma_{j}$ of Eq. (3). Let $\Lambda_{j}=\operatorname{diag}\left(\lambda_{j 1}, \cdots, \lambda_{j d}\right)$ be the diagonal matrix of the eigenvalues of $\Sigma_{j}$, where $\lambda_{j i}$ are the eigenvalues corresponding to the eigenvectors $\psi_{j i}(i=1, \cdots, d)$. We have

$$
\Psi_{j}^{t} \Sigma_{j} \Psi_{j}=\Lambda_{j} .
$$

If the covariance matrix $\Sigma_{j}$ is non-singular, all the corresponding eigenvalues are positive. Otherwise, some eigenvalues may be zero. In general, assume that $\lambda_{j i}(i=1, \cdots, d)$ are ranked in order from larger to smaller as follows:

$$
\lambda_{j 1} \geq \cdots \geq \lambda_{j d_{j}}>\lambda_{j\left(d_{j}+1\right)}=\cdots=\lambda_{j d}=0,
$$

where $d_{j}$ is the number of non-zero eigenvalues of the covariance matrix $\Sigma_{j}$.

Recently, a perturbation approach has been proposed [3. However, for practical application problems, the dimension $d$ may be too high to obtain all the $d$ eigen-vectors.

\subsection{Novel Perturbation Approach}

Assume that all the eigen-vectors corresponding to non-zero eigenvalues are available. Let

$$
z=\left(\psi_{11}, \cdots, \psi_{1 d_{1}}, \cdots \cdots, \psi_{c 1}, \cdots, \psi_{c d_{c}}\right)^{t} x .
$$

This is a linear transformation from the original $d$-dimensional $x$ space to a new $\bar{d}$-dimensional $z$ space, where

$$
\bar{d}=\sum_{j=1}^{c} d_{j} .
$$

Suppose

$$
\bar{d}<d .
$$

Thus, the new $z$ space can be regarded as a "compact" space of the original $x$ space. Instead of the Bayes classifier of Eq. (2) in $x$ space, a Bayes classifier can be introduced in the $z$ space

$$
x \rightarrow \omega_{i} \text { with } i=\arg \max _{j} p\left(z \mid \omega_{j}\right) .
$$

Obviously, $p\left(z \mid \omega_{1}\right)$ has formally a Gaussian distribution since the transformation of Eq. (88) is linear. We are going to propose a novel perturbation approach to determine $p\left(z \mid \omega_{1}\right)$ in the rest of this section.

Conditional Distribution $\boldsymbol{p}\left(\boldsymbol{z} \mid \boldsymbol{\omega}_{1}\right)$. We know that $\left(\psi_{11}, \cdots, \psi_{1 d_{1}}\right)$ are eigenvectors corresponding to the non-zero eigenvalues of the covariance matrix $\Sigma_{1}$. In general, the $\bar{d}-d_{1}$ eigen-vectors $\left(\psi_{21}, \cdots, \psi_{2 d_{2}}, \cdots, \psi_{c 1}, \cdots, \psi_{c d_{c}}\right)$ are not the eigen-vectors corresponding to zero eigenvalues of the covariance matrix $\Sigma_{1}$. 
Firstly, let

$$
\left(\xi_{1}, \cdots, \xi_{\bar{d}}\right) \Leftarrow\left(\psi_{11}, \cdots, \psi_{1 d_{1}}, \psi_{21}, \cdots, \psi_{2 d_{2}}, \cdots \cdots, \psi_{c 1}, \cdots, \psi_{c d_{c}}\right) .
$$

Then, perform the Gram-Schmit orthogonalization for each $j(j=2, \cdots, \bar{d})$ as follows.

$$
\begin{aligned}
& \xi_{j} \Leftarrow \xi_{j}-\sum_{i=1}^{j-1}\left(\xi_{j}^{t} \xi_{i}\right) \xi_{i}, \\
& \xi_{j} \Leftarrow \xi_{j} /\left\|\xi_{j}\right\| .
\end{aligned}
$$

Giving that $\left(\psi_{11}, \cdots, \psi_{1 d_{1}}, \psi_{21}, \cdots, \psi_{2 d_{2}}, \cdots \cdots, \psi_{c 1}, \cdots, \psi_{c d_{c}}\right)$ has a rank of $\bar{d}$, that is, these eigen-vectors are linearly independent, the Gram-Schmit orthogonalization of Eqs. (12,14) is a linear transformation

$$
\left(\xi_{1}, \cdots, \xi_{\bar{d}}\right)=A\left(\psi_{11}, \cdots, \psi_{1 d_{1}}, \cdots \cdots, \psi_{c 1}, \cdots, \psi_{c d_{c}}\right),
$$

where $A$ is a non-singular upper triangle $\bar{d} \times \bar{d}$ matrix.

Theorem 1. Let

$$
y=\left(\xi_{1}, \cdots, \xi_{\bar{d}}\right)^{t} x .
$$

The covariance matrix of $p\left(y \mid \omega_{1}\right)$ is a diagonal matrix

$$
\operatorname{diag}\left(\lambda_{11}, \cdots, \lambda_{1 d_{1}}, 0, \cdots \cdots, 0\right) .
$$

The proof of Theorem 1 is omitted here.

Denote the diagonal elements of the covariance matrix in Eq. (17) as $\bar{\lambda}_{i}(i=$ $1, \cdots, \bar{d})$. By changing the zero-diagonal elements of the covariance matrix in Eq. (17) with a perturbation factor $\varepsilon$, that is

$$
\bar{\lambda}_{d_{1}+1}=\cdots=\bar{\lambda}_{\bar{d}}=\varepsilon,
$$

we can determine $p\left(y \mid \omega_{1}\right)$ as follows

$$
p\left(y \mid \omega_{1}\right)=\prod_{i=1}^{\bar{d}} \frac{1}{\left(2 \pi \bar{\lambda}_{i}\right)^{\frac{1}{2}}} \exp \left[-\frac{\left(y_{i}-\bar{\mu}_{i}\right)^{2}}{2 \lambda_{i}}\right],
$$

where

$$
\bar{\mu}_{i}=\xi_{i}^{t} \mu_{1}(i=1, \cdots, \bar{d}) .
$$

From Eqs. (8), (15) and (16), we have

$$
z=A^{-1} y \text {. }
$$

Then, a novel perturbation approach to determine $p\left(z \mid \omega_{1}\right)$ can be proposed

$$
p\left(z \mid \omega_{1}\right)=p\left(y \mid \omega_{1}\right)\left|A^{-1}\right|,
$$

where $\left|A^{-1}\right|$ is the determinant of the inverse matrix of $A$. 
Conditional Distribution $\boldsymbol{p}\left(\boldsymbol{z} \mid \boldsymbol{\omega}_{\boldsymbol{j}}\right)$. It is now ready to propose an algorithm to determine the conditional distribution $p\left(z \mid \omega_{j}\right)(j=2, \cdots, c)$.

Step 1. Initialize $\left(\xi_{1}, \cdots, \xi_{\bar{d}}\right)$ firstly by assigning $d_{j}$ eigen-vectors of the covariance matrix $\Sigma_{j}$ and then by assigning all the other $\bar{d}-d_{j}$ eigen-vectors of the covariance matrix $\Sigma_{i}(i \neq j)$, that is,

$$
\left(\xi_{1}, \cdots, \xi_{\bar{d}}\right) \Leftarrow\left(\psi_{j 1}, \cdots, \psi_{j d_{j}}, \psi_{11}, \cdots, \psi_{1 d_{1}}, \cdots \cdots, \psi_{c 1}, \cdots, \psi_{c d_{c}}\right) .
$$

Step 2. Perform the Gram-Schmit orthogonalization according to Eqs. (13) and (14). Thus, we obtain the matrix $A$ in Eq. (15).

Step 3. Substitute $\left(\lambda_{j 1}, \cdots, \lambda_{j d_{j}}\right)$ for $\left(\lambda_{11}, \cdots, \lambda_{1 d_{1}}\right)$ in Eq. (17). Substitute $d_{j}$ for $d_{1}$ in Eq. (18). Substitute $\mu_{j}$ for $\mu_{1}$ in Eq. (20). Thus, we can obtain the conditional distribution $p\left(y \mid \omega_{j}\right)$ by performing the transformation of Eq. (16) and substituting $\omega_{j}$ for $\omega_{1}$ in Eq. (19).

Step 4. Obtain the conditional distribution $p\left(z \mid \omega_{j}\right)$ by substituting $\omega_{j}$ for $\omega_{1}$ in Eq. (22).

\section{Experiments}

In this section, experiments for face analysis have been performed on CMU facial expression image database to test the effectiveness of the proposed PCA-based Bayes classifier.

From CMU-Pittsburgh AU-Coded Facial Expression Database 4, 312 facial expression mask images can be obtained by using a spatial adaptive triangulation technique based on local Gabor filters [5]. Six facial expressions are concerned as follows: anger, disgust, fear, joy, unhappy, and surprise. For each expression, there are 52 images with a resolution of $55 \times 59$, the first 26 images of which have moderate expressions while the last 26 images of which have intensive expressions.

In experiments, for each expression, the first $k(k=5,10,15,20,25)$ images are for training and all the other images are for test. Experiments have been performed by using the proposed PCA-based Bayes classifier and the minimum distance classifier, respectively. Experimental results with different $k$ are listed in Table 1 .

From Table 1, we can see that the proposed PCA-based Bayes classifier performs obviously better than the minimum distance classifier. As the number of

Table 1. Classification rates on CMU facial expression image database

\begin{tabular}{|c|c|c|c|}
\hline Images & $\mathrm{k}$ & Minimum distance & PCA-based Bayes \\
\hline \hline \multirow{4}{*}{$55 \times 59$} & 5 & $25.53 \%$ & $27.30 \%$ \\
\cline { 2 - 4 } & 10 & $29.76 \%$ & $63.89 \%$ \\
\cline { 2 - 4 } & 15 & $50.45 \%$ & $73.87 \%$ \\
\cline { 2 - 4 } & 20 & $59.38 \%$ & $88.02 \%$ \\
\cline { 2 - 4 } & 25 & $64.02 \%$ & $95.68 \%$ \\
\hline
\end{tabular}


training samples $k$ increases, the classification rate by using the proposed classifier increases much faster than that by using the minimum distance classifier. It means that the proposed classifier can perform much more efficient than the minimum distance classifier.

\section{Conclusions}

In this paper, we have proposed a novel PCA-based Bayes classifier in high dimensional spaces. Experiments for face analysis have been performed on CMU facial expression image database. It is shown that the proposed classifier performs much better than the minimum distance classifier. With the proposed classifier, we can not only improve the classification rate, but also obtain a better understanding of data.

\section{Acknowledgements}

This work was supported by Ramón y Cajal research fellowship from the Ministry of Science and Technology, Spain and the National Natural Science Foundation of China under Grant No. 60473039.

\section{References}

1. K. Fukunaga. Introduction to Statistical Pattern Recognition. Academic Press, 1990.

2. Zhong Jin, Jingyu Yang, Zhongshan Hu, and Zhen Lou. Face recognition based on the uncorrelated discriminant transformation. Pattern Recognition, 34(7):1405-1416, 2001.

3. Z. Jin, F. Davoine, and Z. Lou. An effective EM algorithm for PCA mixture model. In Structural, Syntactic and Statistical Pattern Recongnition, volume 3138 of Lecture Notes in Computer Science, pp. 626-634, Lisbon, Portugal, Aug. 18-20 2004. Springer.

4. Takeo Kanade, Jeffrey F. Cohn, and Yingli Tian. Comprehensive database for facial expression analysis. In Proceedings of the Fourth International Conference of Face and Gesture Recognition, pages 46-53, Grenoble, France, 2000.

5. S. Dubuisson, F. Davoine, and M. Masson. A solution for facial expression representation and recognition. Signal Processing: Image Communication, 17(9):657-673, 2002. 